\title{
Hypoxic Pretreatment Adipose-derived Stem Cell Exosomes Improve Cognition by Delivery Circ-Epc1 and Shifting Microglial M1/M2 Polarization in Alzheimer's Disease Mice Model
}

Haining Liu

Shanghai Mental Health Center

Mingming Jin ( $\nabla$ asdjinmingming@126.com )

Shanghai University of Medicine and Health Sciences

Minxiu Ji

Shanghai Mental Health Center

Wei Zhang

Shanghai Mental Health Center

An Liu

Shanghai Mental Health Center

Tao Wang

Shanghai Mental Health Center

\section{Research}

Keywords: circ-Epc1, alzheimer's disease, adipose derived stem cells, exosomes, microglia

Posted Date: August 27th, 2021

DOl: https://doi.org/10.21203/rs.3.rs-815669/v1

License: (c) (1) This work is licensed under a Creative Commons Attribution 4.0 International License.

Read Full License 


\section{Abstract}

Background: Alzheimer's disease (AD) is the most major dementia in the globe. Increasing evidence informs that exosomes from hypoxic pretreatment adipose-derived stem cells (ADSCs) could therapeutically affect cognitive function in AD-associated pathophysiology. However, their role and regulatory mechanism remain largely unknown.

Methods: High-throughput sequencing was used to identify differentially expressed exosomal circRNAs from ADSCs or hypoxia pretreated ADSCs. Luciferase reporter assays and RT-qPCR were used to investigate the relationships between circ-Epc1, miR-770-3p, and TREM2. APP/PS1 double transgenic AD model mice were then utilized to study therapeutic effect regarding circ-Epc1 in ADSCs exosomes. BV2 cells were used to understand the regulatory relationship between circ-Epc1, miR-770-3p, and TREM2 and how these interactions modulated phenotypic transformation and inflammatory cytokine expression in microglia.

Results: The result show that exosomes from hypoxia pretreatment ADSCs had a greater therapeutic effect at improving cognitive function by decreasing neuronal damage in the hippocampus. Highthroughput sequencing found that circ-Epc1 played an important role in hypoxia pretreated ADSC exosomes regarding their ability to improve cognitive function. Luciferase reporter assays showed that TREM2 and miR-770-3p were downstream targets of circ-Epc1. Overexpressing miR-770-3p or downregulating TREM2 reversed the effects of circ-Epc1 on M2 microglia under LPS treatment. In vivo experiments showed that circ-Epc1-containing ADSC exosomes increased the therapeutic effect of exosome at improving cognitive function by decreasing neuronal damage and shifting hippocampal microglia from M1 to M2 polarization.

Conclusions: Taken together, the data found that hypoxic pretreatment ADSCs exosomes improve cognition by delivery circ-Epc1 and shifting microglial M1/M2 polarization in alzheimer's disease mice model.

\section{Introduction}

Alzheimer's disease (AD) is the most common contribution regarding dementia in the globe and is being more prevalent due to human aging population; it thus constitutes a big challenge to health care systems $[1,2]$. The hallmark pathological feature of $A D$ is the deposition of $\beta$-amyloid $(A \beta)$, which has strong neurotoxicity in brain tissue and leads to cognitive impairment $[3,4] . A D$ is a microglia-mediated neuroinflammatory disease. More evidence inferrs that microglial activation in central nervous system is heterogeneous, which could be categorized to disparate classes: the M1 and M2 phenotypes. Due to the phenotype activated, microglia could generate either neuroprotective or cytotoxic effects [5]. The different microglial phenotypes vary according to disease stage and severity. The ability to control stage-specific switching of M1/M2 phenotypes in appropriate time windows might supply therapeutic benefits to $A D$ patients $[6,7]$. 
Mesenchymal stem cells (MSCs) are a family of adult stem cell that could produce a large number of multivesicular bodies that are secreted in the form of exosomes. Exosomes vary in size, with diameters of approximately 30-150 nm, which can cross blood-brain barrier. Exosomes can carry a large number of non-coding (nc) RNAs including circRNAs to the brain to alter physiology. Exosomes derived from MSCs play regulatory roles on $A D$ [8-10]. A former investigation illustrated that when adipose-derived stem cell (ADSC) exosomes were injected intravenously, they improved learning and memory capabilities significantly, which decremented plaque deposition and $A \beta$ levels to normalize inflammatory cytokine levels [11]. Present study discovered that hypoxia pretreated ADSC exosomes had increased treatment efficacy for AD-induced nerve damage and cognitive impairment in the hippocampus. The subsequent aim of current study was to illustrate the role and regulatory mechanism regarding ADSC exosomes on $A D$.

\section{Materials \& Methods}

\section{Animals and ethics statement}

We obtained APP/PS1 double transgenic mice and B6C3-Tg (APPswe, PSEN1dE9) 85Dbo/J (original species No. 004462) from Jax Laboratory (Bar Harbor, ME, USA). Our lab maintained transgenic mice on a standard 12-h light/dark cycle under constant temperature with free access to water and food. Animal ethics committee in Shanghai Mental Health Center, Shanghai Jiao Tong University School of Medicine approved this study. All protocols followed "Guide for the Care and Use of Laboratory Animals" from National Institutes of Health (Bethesda, MD, USA), and we made all efforts to minimize the number of animals utilized and any discomfort experiments.

\section{ADSCs isolation, culture, and identification}

Our lab washed adipose tissue harvested from healthy people or normal mice with phosphate-buffered saline (PBS) and minced it before digestion with $0.2 \%$ collagenase I (Sigma-Aldrich, St. Louis, MO, USA) for $1 \mathrm{~h}$ at $37^{\circ} \mathrm{C}$ with intermittent shaking. Technician washed tissue that digested with Dulbecco's Modified Eagle's Medium (DMEM; Sigma-Aldrich) including fetal bovine serum (FBS, Gibco BRL, Frederick, MD, USA) of $15 \%$, and centrifuged it at $1000 \mathrm{rpm}$ for $10 \mathrm{~min}$ to eliminate mature adipocytes. Our lab resuspended pellet in DMEM supplied with FBS of $15 \%, 100 \mathrm{U} / \mathrm{mL}$ penicillin, and $100 \mu \mathrm{g} / \mathrm{mL}$ streptomycin and cultured it at $37^{\circ} \mathrm{C}$ with $\mathrm{CO}_{2}$ of $5 \%$. When the ADSCs had reached $80 \% \sim 90 \%$ confluency, our lab detached them with $0.02 \%$ ethylenediaminetetraacetic acid (EDTA) $/ 0.25 \%$ trypsin (Sigma-Aldrich) for $5 \mathrm{~min}$ at room temperature and then replated them. For phenotypic analyses, we leveraged fluorescein isothiocyanate (FITC)-conjugated CD29, CD44, CD90, CD105, and vWF antibodies. An IgG-matched isotype was employed as internal control for every antibody. Normoxic ADSC cultures were in $95 \%$ air $\left(20 \% \mathrm{O}_{2}\right)$ and $\mathrm{CO}_{2}$ of $5 \%$. For hypoxia induction, our lab cultured ADSCs in $94 \% \mathrm{~N}_{2}, 1 \% \mathrm{O}_{2}$, and $\mathrm{CO}_{2}$ of $5 \%$. 


\section{ADSCs multilineage differentiation}

To validate ADSCs multilineage differentiation, third-passage mouse ADSCs were cultured in adipogenic differentiation medium (Sigma-Aldrich), which were stained with oil red $\mathrm{O}$ after $2 \mathrm{w}$, or cultured in osteogenic differentiation medium (Sigma-Aldrich) and stained with alizarin red after $3 \mathrm{w}$.

\section{ADSC-derived exosome isolation and identification}

After reaching $80 \% \sim 90 \%$ confluency, our lab rinsed ADSCs with PBS and cultured them in FBS-free endothelial cell growth medium (EGM)-2MV supplemented with $1 \times$ serum replacement solution (PeproTech, Cranbury, NJ, USA) for another $2 \mathrm{~d}$. We erased conditioned culture medium and centrifuged it at $300 \times g$ for $10 \mathrm{~min}$ and then at $2000 \times g$ for $10 \mathrm{~min}$ to erase apoptotic cells and cellular debris. Briefly, we removed cell debris and large membrane vesicles by sequential centrifugation at $300 \times g$ for $10 \mathrm{~min}$, $2,000 \times g$ for $10 \mathrm{~min}$, and $10,000 \times g$ for $0.5 \mathrm{hr}$, followed by filtration through $0.22-\mu \mathrm{m}$ syringe filters. Afterwards, our lab transferred supernatant that cleared to fresh tube and spun it at 100,000 $\times g$ for 70 min. Then, we completely removed supernatant, and washed the pellet with PBS to collect exosomes. We characterized exosomes via transmission electron microscopy and western blotting, and the size was determined by dynamic light scattering using nanoparticle tracking analysis (NTA; NanoSight, Malvern, Worcestershire, UK).

\section{Exosome tracing}

We labelled exosomes using lipophilic dye Dil (1,1'-dioctadecyl-3,3,3',3'-tetramethylindocarbocyanine perchlorate; Thermo Fisher Scientific, Waltham, MA, USA) for biodistribution detection.

\section{Strand-specific high-throughput RNA-Seq library construction}

Our team extracted total RNA from ADSCs exosomes (Exo) and hypoxia-pretreated ADSC exosomes (HExo) with or without hyperglycemia pretreatment through TRIzol Reagent (Invitrogen, Carlsbad, CA, USA). About $3 \mu$ g total RNA from every sample was subjected to VAHTS Total RNA-seq (H/M/R) Library Prep Kit from Illumina (Vazyme Biotech Co., Ltd, Nanjing, China). In this way, we removed ribosomal RNA and retained other types of RNA including ncRNA and mRNA. Our lab treated purified RNA with $40 \mathrm{U}$ RNase R (Epicenter, Madison, WI, USA) at $37^{\circ} \mathrm{C}$ for $3 \mathrm{~h}$, followed by TRIzol purification. Our lab made RNAseq libraries via KAPA Stranded RNA-Seq Library Prep Kit (Roche, Basel, Switzerland), which were subjected to deep sequencing with an Illumina HiSeq 4000 at Aksomics, Inc. (Shanghai, China).

\section{Exosome injections}


We intravenously treated two-month-old APP/PS1 mice with PBS (AD), exosomes derived from ADSCs (Exo), hypoxia-pretreated ADSCs (HExo), or circ-Epc1-expressing ADSCs (circ-Epc1-Exo) monthly for 2 months ( $n=10$ mice per group). Of all groups, the injection volume was $100 \mu \mathrm{L}$. Exosomes from $1 \times 10^{9}$ ADSCs were dissolved in $100 \mu \mathrm{L}$ PBS. There was no drop-out during normal breeding along with injection period. To detect exosome presence in brain, we euthanized three mice from each group at $5 \mathrm{~h}$ postinjection to examine brain slices by confocal microscopy after counterstaining with 4',6-diamidino-2phenylindole (DAPI).

\section{Morris water maze (MWM) test}

Our lab tested memory and learning function with MWM [12]. Operator blinded to treatment groups performed all examinations. The apparatus consisted of a round steel pool (diameter: $122 \mathrm{~cm}$; height: 60 $\mathrm{cm}$ ) that was filled with water to $1 \mathrm{~cm}$ higher than the platform (diameter: $10 \mathrm{~cm}$; depth: $30 \mathrm{~cm}$ ) top. Blue curtain with cues surrounded the pool, which was put in an isolated room $\left(20^{\circ} \mathrm{C}, 60 \%\right.$ humidity). We maintained water at $21^{\circ} \mathrm{C}$ and opacified it via inserting titanium dioxide.

Our team performed testing for $5 \mathrm{~d}$. The first $4 \mathrm{~d}$ (P40-P43) comprised a place navigation (reference memory) test including 16 training trials ( 4 trials per day for $4 \mathrm{~d}$, with 30-40 min inter-trial interval). At the start of every trial, we put mice in the water facing the wall in various starting locations (south, north, west or east), which were allowed $1 \mathrm{~min}$ to discover and $15 \mathrm{~s}$ to stay on top of the hidden platform. If the mouse could not locate the platform within $1 \mathrm{~min}$, it was guided to and allowed to stay on the platform for $15 \mathrm{~s}$. We employed video tracking system to track swimming activity regarding every mouse. Escape latency, say, timing from placement into the water to staying on the platform, was tracked. We performed spatial probe test where the platform was moved out of the pool. We placed the animal in opposite quadrant and allowed it to swim freely for $2 \mathrm{~min}$. We tracked platform crossing numbers. Our team analyzed data via motion-detection software for MWM test (Shanghai Mobile Datum Information Technology Co., Shanghai, China).

\section{BV2 cell culture and transfection}

Our lab maintained BV2 cells (Wuhan Biofavor Biotechnology Service Co., Ltd., Wuhan, China) in DMEM (Invitrogen) supplemented with FBS (Invitrogen) of $10 \%$ under atmosphere containing $5 \% \mathrm{CO}_{2} / 95 \%$ air. For phenotypic analyses, we transfected BV2 cells with a small interfering (si)RNA against TREM2, a circEpc1 overexpression plasmid, or a miR-770-3p overexpression plasmid (mimic) (GeneCopoeia, Shanghai, China) through Lipofectamine 2000 (Invitrogen) following standard procedures. We used cells for further experimentation after $2 \mathrm{~d}$ and exposed them to LPS $(1 \mu \mathrm{g} / \mathrm{mL})$ for $1 \mathrm{~d}$ before phenotypic analysis.

\section{RNA and miRNA extraction with real-time (RT)-PCR}


Our lab isolated total RNA from serum, cells, or brain tissues via TRIzol reagent. We synthesized first strand cDNA through PrimeScript RT Master Mix (Perfect Real Time) Kit (RR036A, Shiga, Japan), which was leveraged for RT-PCR, along with reverse and forward primers and Power SYBR Green PCR Master Mix (Life Technologies, Carlsbad, CA, USA). U6 and GAPDH were utilized as internal controls. We analyzed data via $2^{-\triangle \Delta C t}$ method.

\section{Luciferase reporter assays}

We cloned putative miR-770-3p binding site in 3'-UTR of target gene TREM2 and circ-Epc1 (Wt or Mut) into psi-CHECK vector (Promega, Madison, WI, USA) downstream of firefly luciferase 3'-UTR or circ-Epc1 as primary luciferase signal with Renilla luciferase as normalization signal, these vectors were termed TREM2-Wt/circ-Epc1-Wt and TREM2-Mut/circ-Epc1-Mut, respectively. psi-CHECK vector provided Renilla luciferase signal as normalization to compensate the differences between transfection and harvesting efficiencies. We performed transfection into HEK293 cells through Lipofectamine 2000 (Invitrogen). Our lab detected Renilla and firefly luciferase activities $1 \mathrm{~d}$ after transfection with Dual-Luciferase Reporter Assay System (Promega) through luminometer (Molecular Devices, San Jose, CA, USA). Our lab detected relative Renilla luciferase activities following the manufacturer (Promega) instructions.

\section{Immunohistochemistry (IHC) and immunofluorescence (IF) analyses}

Our team fixed brain tissue samples in $10 \%$ formalin solution, embedded them in paraffin, which were sectioned at $5 \mu \mathrm{m}$. Our lab stained tissue sections with TUNEL detection kit (Zeiss, Oberkochen, Germany) for apoptosis evaluation. Our lab performed IF staining for Iba-I, CD11b, and CD206 to validate microglial polarization. We analyzed results through Axiophot light microscope (Zeiss) or a fluorescence microscope (Nikon, Tokyo, Japan), which were photographed with digital camera.

\section{Enzyme-linked immunosorbent assay (ELISA)}

We collected cell culture medium after the treatments that mentioned above. We utilized ELISA kits (R\&D Systems, Minneapolis, MN) to obtain the interleukin (IL)-1 $\beta$, IL-6, and tumor necrosis factor (TNF)-a levels following the instructions.

\section{Statistical analysis}

Continuous variations are denoted as mean \pm standard deviation (SD). We used one-way analysis of variance for multiple comparisons via GraphPad Prism (GraphPad Software, Inc., La Jolla, CA, USA). A Pvalue $\leq 0.05$ inferred statistically significance. 


\section{Results}

\section{ADSC-exosomes characterization}

Former investigations reported that exosomes from ADSCs reduce $A \beta$ pathology and neuronal cell apoptosis in a transgenic mouse model of $A D$ [13]. Nevertheless, the underlying mechanism is unclear. In current study, our lab isolated ADSCs to confirm the typical cobblestone-like morphology (Fig. 1A). IF staining was positive for mesenchymal cell markers CD90, CD29, CD44, and CD105, and negative for endothelial marker vWF (Fig. 1B-G). Oil-red-O (Fig. 1H) and alizarin red (Fig. 1I) staining data validated that ADSCs could differentiate into various lineages such as osteoblasts and adipocytes.

Western blot analysis of exosome lysates demonstrated positive expression regarding exosomal proteins CD81 and CD63 in both normal and hypoxia-pretreated ADSCs (Fig. 1J). Transmission electron microscopy illustrated that ADSC-exosomes exhibited characteristic cup-shaped morphology (Fig. 1K). We quantified size of ADSC-exosomes by Zetasizer Nano. The mean vesicle diameter was $80-130 \mathrm{~nm}$ (Fig. 1L), which was consistent with exosomes that formerly described [14]. The data inferred that the nanoparticles were exosomes.

\section{Exosomes from hypoxia-pretreated ADSCs improved cognitive function by decreasing neuronal damage in the hippocampus.}

To calculate exosome presences derived from ADSCs in cortex and hippocampus, we observed brain slices under a fluorescence microscope $5 \mathrm{~h}$ after injection. We discovered Dil-labeled exosomes in hippocampus and cortex (Fig. 2A). ELISA detection show inflammatory factor IL-6, TNF-a, and IL-1 $\beta$ expressions in brain tissue. These results showed that exosome treatment inhibited inflammatory factor expression. Exosomes from hypoxia-pretreated ADSCs (HExo) had more therapeutic effect and decreased IL-6, TNF-a, and IL-1 $\beta$ expression to a greater extent (Fig. 2B-D). IHC show that HExo suppressed nerve apoptosis in the hippocampus (Fig. 2E-F) more than other treatments. To explore behavioral consequences of exosomes in the mouse model of $A D$, we assessed spatial learning and memory with MWM (Fig. 2G). Exo treatment decreased escape latency more than the untreated AD mice. HExo had a greater therapeutic effect in decreasing escape latency than normal exosomes. Furthermore, during spatial probe test, the platform crossing number decremented in Exo treatment group, particularly in the HExo treatment group (Fig. 2H).

\section{The greater therapeutic effect of hypoxia-pretreated ADSC exosomes at improving cognitive function involves circ- Epc1.}


Former investigations have found that ncRNAs function importantly in AD pathogenesis [15]. Present study utilized high-throughput sequencing to explore the differentially expressed circRNAs in Exo and HExo. The results showed that hypoxia pretreatment lead to abnormal expression of circRNAs (Fig. 3A). Compared with Exo, hypoxia pretreatment lead to 3,133 circRNAs being upregulated and 1,554 circRNAs downregulated (Fig. 3B). RT-qPCR detection show upregulated expression of 10 circRNAs (circ-Rtn4, circHipk2, Circ-Epc1, circ-Asxl1, circ-Atp9b, circ-Xrn2, circ-Sass6, circ-Chd7, circ-Taf4a, and circ-Prdm2) between Exo and HExo. These data demonstrated that only circ-Epc1 expression significantly increased in HExo compared with Exo (Fig. 3C). Bioinformatics analysis advised that circ-Epc1 was derived and cyclized from an exon of Epc1, which is located at chr18:6448902-6450637. Together, these data suggested that circ-Epc1 may play a role in improving cognitive function.

\section{TREM2 and miR-770-3p are downstream targets of circ- Epc1.}

A previous study found that circRNAs can regulate gene expression by sponging miRNAs [16]. Bioinformatics analysis advised that circ-Epc1 could interact with miRNAs including miR-344b-5p, miR346-3p, miR-106a-5p, miR-20a-5p, miR-93-5p, miR-17-5p, miR-467a-5p, miR-290a-3p, mir-293-3p, miR-877$5 p$, miR-434-5p, miR-770-3p, and miR-296-5p. Next, we constructed luciferase reporter vectors containing the circ-Epc1 sequence. We then transfected different miRNA mimics into HEK293 cells. Data illustrated that only miR-770-3p could significantly decrease fluorescein intensity, advising that miR-770-3p was a circ-Epc1 downstream target (Fig. 4A). Luciferase reporter analysis further confirmed that miR-770-3p inhibited luciferase activity in wild-type (WT) cells but not in mutated (MUT) cell lines (Fig. 4B-C), suggesting that miR-770-3p was the circ-Epc1 target.

Bioinformatics analysis found that TREM2 was a miR-770-3p downstream target. To further verify correlation regarding miR-770-3p and TREM2, WT or MUT 3'-UTR-TREM2 sequences including miR-770$3 p$ binding sequence were inserted into luciferase reporter vector (Fig. 4D). Our team transfected luciferase reporter vector into 293T cells with or without miR-770-3p mimic. This luciferase reporter analysis discovered that miR-770-3p inhibited luciferase activity in WT cells, but not in cell lines with the MUT sequence (Fig. 4E), advising that TREM2 was miR-770-3p target. RT-qPCR detection showed that circ-Epc1 expression incremented in BV2 cells after transfection with a circ-Epc1 overexpression vector, while a miR-770-3p overexpression vector or siRNA against TREM2 could not reverse circ-Epc1 expression (Fig. 4F). Overexpressing circ-Epc1 inhibited miR-770-3p expression, but transfecting miR-770-3p mimics reversed and promoted miR-770-3p expression. Transfecting siRNA against TREM2 did not rescue miR770-3p expression after silencing circ-Epc1 (Fig. 4G). Our results also showed that overexpressing circEpc1 promoted TREM2 expression, while overexpressing miR-770-3p reversed this effect and promoted circ-Epc1 (Fig. 4H). Together, these data showed that TREM2 and miR-770-3p were important downstream targets of circ-Epc1 and that circ-Epc1 may regulate TREM2 by sponging miR-770-3p. 


\section{Overexpressing miR-770-3p or downregulating TREM2 reversed the effect of circ-Epc1 on shifting M2 microglial under LPS stimulation.}

A previous study found that counteracting immunomodulation of microglia polarization could reduce cognitive aging impairment and AD [17]. In this study, BV2 cells were transfected with circ-Epc1 overexpression vector, miR-770-3p mimic, or si-TREM2 individually or in combination. After exposure to 1 $\mu \mathrm{g} / \mathrm{mL}$ LPS for $1 \mathrm{~d}$, the we collected BV2 cells for phenotypic analysis. IF detection of microglial/macrophage polarization was performed using lba-I (total microglia), CD11 b ${ }^{+}$(M1 microglia), and CD206 ${ }^{+}$(M2I microglia). The results showed that upregulating circ-Epc1 decreased M1 microglial differentiation by decreasing CD11b expression. In contrast, overexpressing miR-770-3p or downregulating TREM2 partially reversed M1 microglial differentiation following circ-Epc1 overexpression. We also found that upregulating circ-Epc1 promoted M1 microglial differentiation by increasing CD11b expression. Additionally, overexpressing miR-770-3p or silencing TREM2 partially reversed M2 microglial differentiation in circ-Epc1-overexpressing cells (Fig. 5A-D). ELISA detection showed that miR-770-3p overexpression or silencing TREM2 partially reversed the inhibitory effect of circEpc1 to the inflammatory factor expressions such like IL-6, TNF-a, and IL-1 $\beta$ (Fig. 5E-G).

\section{Circ-Epc1 containing ADSCs exosomes (circ-Epc1-Exo) increased therapeutic efficacy and improved cognitive function by decreasing neuronal damage and shifting microglia from M1 to M2 in the hippocampus.}

To illustrate the protective effect of circ-Epc1 on cognitive function, APP/PS1 double transgenic mice received PBS, HExo, or circ-Epc1-Exo treatment. IHC showed that circ-Epc1-Exo treatment had a greater therapeutic effect than HExo at decreasing hippocampal apoptosis (Fig. 6A-B). To elucidate the behavioral consequences of exosome treatment in the mouse model of $A D$, our lab assessed spatial learning and memory via MWM (Fig. 6C). Circ-Epc1-Exo treatment had a greater therapeutic effect than HExO at decreasing escape latency compared with untreated $A D$ mice. Furthermore, during spatial probe test, the platform crossing number decremented in HExo treatment group, particularly in the circ-Epc1-Exo treatment group (Fig. 6D).

IF detection of microglial/macrophage polarization in the hippocampus was performed using Iba-I (total $^{+}$

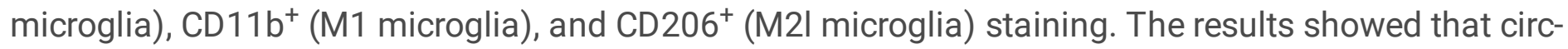
Epc1-Exo treatment had a greater therapeutic effect than HExo at decreasing M1 microglial differentiation and promoting M2 microglial differentiation by decreasing CD11b and increasing CD206 expression (Fig. 6E-H). 
ELISA detection showed that circ-Epc1-Exo treatment had considerable therapeutic effect than HExo on the inflammatory factor expressions such like TNF- $\alpha$, IL-1 $\beta$ and IL-6 (Fig. 6I-K). RT-qPCR detection showed that circ-Epc1-Exo treatment also had a greater therapeutic effect than HExo at increasing miR-770-3p and decreasing TREM2 expression in hippocampal tissues (Fig. 6L-M).

\section{Discussion And Conclusions}

The AD prevalence has incremented in recent years, leading to heavy burden regarding economies, families, and the whole society. Currently, there are no efficient therapeutic drugs for AD. Stem cell exosome therapy is a preferrable non-pharmacotherapy treatment because of its wide positive effect range, fewer side effects, and low economic burden [18-20]. Previous studies have found that ADSC exosome treatment can reduce $A \beta 40$ and $A \beta 42$ levels together with the $A \beta 42 / 40$ ratio of $A D$ cells [13]. Current study suggested that hypoxia-pretreated ADSC exosome treatment had a greater therapeutic effect than ADSC exosomes at decreasing apoptosis and inflammatory cytokines in the hippocampus. Cognitive function tests found that hypoxia-pretreated ADSC exosome treatment had greater effects than treatment with $A D S C$ exosomes at recovering $A D$-induced cognitive impairment.

To verify the regulatory mechanism that underlined how ADSC exosomes alter AD pathophysiology, we produced high-throughput sequencing of exosomes from different treatment groups. The results showed that circRNAs were abnormally expressed in exosomes from hypoxia-pretreated ADSCs when compared with untreated ADSC exosomes. RT-qPCR detection showed that circ-Epc1 levels were significantly increased in hypoxia-pretreated ADSC exosomes. Circ-Epc1 is derived and cyclized from part of the Epc1 gene, which is located at chr18:6448902-6450637. Overall, our data suggested that circ-Epc1 may play a role in improving cognitive function.

CircRNAs are a particular family of noncoding single-stranded highly stable ribonucleic acid molecules that are abundant in eukaryotic transcriptome. CircRNAs are enriched significantly in human retinal and brain tissues [15]. Previous studies have found that circRNAs contribute to AD pathogenesis [21, 22]. Another study found that circRNAs regulate gene expression by sponging miRNAs [23]. In this study, we found that circ-Epc1 interacted with miR-770-3p using a luciferase reporter system. The literature reports that miR-770-3p expression significantly increases with aging [24]. MiR-770-3p expression can facilitate apoptosis by inducing inflammation [25]. Further studies found that miR-770-3p interacts with triggering receptor that expressed in myeloid cells 2 (TREM2), which was also verified by our luciferase reporter assays. TREM2 is a receptor only expressed by microglia in brain, where it changes microglial immune homeostasis. Human genetic investigations demonstrated that loss-of-function mutations in TREM2 signaling are highly associated with elevated risk of age-related neurodegenerative traits such like AD. TREM2 loss confers resilience to synaptic and cognitive impairment in aged mice [26]. TREM2 also ameliorates neuroinflammatory responses and cognitive impairment via the PI3K/AKT/FoxO3a pathway in $A D$ mice [27]. 
In this study, the data found that overexpressing circ-Epc1 promoted TREM2 and decreased miR-770-3p expression. Overexpressing miR-770-3p also decreased TREM2 expression. TREM2 overexpression did not influence circ-Epc1 or miR-770-3p expression, suggesting that TREM2 and miR-770-3p were downstream targets of circ-Epc1. Circ-Epc1 regulated TREM2 by sponging miR-770-3p. Increasingly, studies are finding that $A D$ induces microglial activation and transforms their polarization towards the M1 phenotype, which is associated with the production of proinflammatory cytokines, eventually leading to nerve cell damage in the hippocampus and cognitive impairment [28-30]. In our study we found that circ-Epc1 expression promoted M2 microglial phenotypes under LPS stimulation. However, overexpressing miR-770-3p or downregulating TREM2 reversed the promotion effects of circ-Epc1 on shifting the M2 microglial phenotype under LPS. Our in vivo experiments also found that circ-Epc1containing ADSC exosomes partially rescued AD-induced cognition impairment by shifting the microglial phenotype from $M 1$ to $M 2$, which resulted in decreased expression of inflammatory cytokines and apoptosis of hippocampal neurons.

\section{Conclusions}

Taken together, we found that hypoxic pretreatment adipose-derived stem cell exosomes improve cognition by delivery circ-Epc1 and shifting microglial M1/M2 polarization in alzheimer's disease mice model. Further work is necessary to verify and expand the findings, which might ultimately enable us to completely elucidate the AD mechanisms.

\section{Abbreviations}

$A D$, alzheimer's disease; ADSCs, adipose derived stem cell; circRNAs, circular RNAs; $A \beta$, $\beta$-amyloid; FBS, fetal bovine serum; MSCs, mesenchymal stem cells; PBS, phosphate-buffered saline.

\section{Declarations}

\section{Ethical Approval and Consent to participate}

Not applicable.

\section{Consent for publication}

All authors agree to publish the article.

\section{Availability of data and materials}

All data in this study were availabe. 


\section{Competing interests}

The authors declare that they have no competing interests.

\section{Funding}

This work was supported by grants from national Natural Science Foundation of China (81571298), Shanghai Health System Excellent Talent Training Program (2017BR054), Clinical Research Center, Shanghai Mental Health Center (CRC2019ZD03, CRC2017ZD02).

\section{Authors' contributions}

MJ and TW designed the studies and prepared the manuscript with comments from all authors. HL and MJ performed all the experiments and analyzed the data. WZ and AL carried out in all experiments and revised the manuscript. All authors read and approved the final manuscript.

\section{Acknowledgements}

Not applicable.

\section{Authors' information}

${ }^{a}$ Department of Geriatric Psychiatry, Shanghai Mental Health Center, Shanghai Jiao Tong University School of Medicine, Shanghai, 200030, China.

${ }^{b}$ Alzheimer's Disease and Related Disorders Center, Shanghai Jiao Tong University, Shanghai, 200030, China.

'Shanghai Key Laboratory of Molecular Imaging, Shanghai University of Medicine and Health Sciences, Shanghai 201318, P.R. China.

\section{References}

1. Idda ML, Munk R, Abdelmohsen K, Gorospe M. Noncoding RNAs in Alzheimer's disease, Wiley Interdiscip Rev RNA, 9 (2018).

2. Wang Z, Xu P, Chen B, Zhang Z, Zhang C, Zhan Q, Huang S, Xia ZA, Peng W. Identifying circRNAassociated-ceRNA networks in the hippocampus of Abeta1-42-induced Alzheimer's disease-like rats using microarray analysis. Aging. 2018;10:775-88. 
3. Cui MY, Lin Y, Sheng JY, Zhang X, Cui RJ, Exercise Intervention Associated with Cognitive Improvement in Alzheimer's Disease, Neural Plast, 2018 (2018) 9234105.

4. Kirova AM, Bays RB, Lagalwar S, Working memory and executive function decline across normal aging, mild cognitive impairment, and Alzheimer's disease, Biomed Res Int, 2015 (2015) 748212.

5. Tang Y, Le W. Differential Roles of M1 and M2 Microglia in Neurodegenerative Diseases. Mol Neurobiol. 2016;53:1181-94.

6. Maezawa I, Nguyen HM, Di Lucente J, Jenkins DP, Singh V, Hilt S, Kim K, Rangaraju S, Levey Al, Wulff $\mathrm{H}$, Jin LW, Kv1.3 inhibition as a potential microglia-targeted therapy for Alzheimer's disease: preclinical proof of concept, Brain, 141 (2018) 596-612.

7. Yao K, Zu HB, Microglial polarization: novel therapeutic mechanism against Alzheimer's disease, Inflammopharmacology, 28 (2020) 95-110.

8. Kang JM, Yeon BK, Cho SJ, Suh YH. Stem Cell Therapy for Alzheimer's Disease: A Review of Recent Clinical Trials. J Alzheimers Dis. 2016;54:879-89.

9. Reza-Zaldivar EE, Hernandez-Sapiens MA, Minjarez B, Gutierrez-Mercado YK, Marquez-Aguirre AL, Canales-Aguirre AA. Potential Effects of MSC-Derived Exosomes in Neuroplasticity in Alzheimer's Disease. Front Cell Neurosci. 2018;12:317.

10. Chakari-Khiavi F, Dolati S, Chakari-Khiavi A, Abbaszadeh H, Aghebati-Maleki L, Pourlak T, Mehdizadeh A, Yousefi M. Prospects for the application of mesenchymal stem cells in Alzheimer's disease treatment. Life Sci. 2019;231:116564.

11. Cui GH, Guo HD, Li H, Zhai Y, Gong ZB, Wu J, Liu JS, Dong YR, Hou SX, Liu JR. RVG-modified exosomes derived from mesenchymal stem cells rescue memory deficits by regulating inflammatory responses in a mouse model of Alzheimer's disease. Immun Ageing. 2019;16:10.

12. Banks AS, McAllister FE, Camporez JP, Zushin PJ, Jurczak MJ, Laznik-Bogoslavski D, Shulman GI, Gygi SP. B.M. Spiegelman, An ERK/Cdk5 axis controls the diabetogenic actions of PPARgamma. Nature. 2015;517:391-5.

13. Lee M, Ban JJ, Yang S, Im W, Kim M. The exosome of adipose-derived stem cells reduces betaamyloid pathology and apoptosis of neuronal cells derived from the transgenic mouse model of Alzheimer's disease. Brain Res. 2018;1691:87-93.

14. Katsuda T, Tsuchiya R, Kosaka N, Yoshioka Y, Takagaki K, Oki K, Takeshita F, Sakai Y, Kuroda M, Ochiya T. Human adipose tissue-derived mesenchymal stem cells secrete functional neprilysinbound exosomes. Sci Rep. 2013;3:1197.

15. Akhter R. Circular RNA and Alzheimer's Disease. Adv Exp Med Biol. 2018;1087:239-43.

16. Panda AC. Circular RNAs Act as miRNA Sponges. Adv Exp Med Biol. 2018;1087:67-79.

17. Pena-Altamira E, Petralla S, Massenzio F, Virgili M, Bolognesi ML, Monti B. Nutritional and Pharmacological Strategies to Regulate Microglial Polarization in Cognitive Aging and Alzheimer's Disease. Front Aging Neurosci. 2017;9:175. 
18. Yang SY, Shan CL, Qing H, Wang W, Zhu Y, Yin MM, Machado S, Yuan TF, Wu T. The Effects of Aerobic Exercise on Cognitive Function of Alzheimer's Disease Patients. CNS Neurol Disord Drug Targets. 2015;14:1292-7.

19. Jiang L, Dong H, Cao H, Ji X, Luan S, Liu J. Exosomes in Pathogenesis, Diagnosis, and Treatment of Alzheimer's Disease. Med Sci Monit. 2019;25:3329-35.

20. Malm T, Loppi S, Kanninen KM. Exosomes in Alzheimer's disease. Neurochem Int. 2016;97:193-9.

21. Lu Y, Tan L, Wang X. Circular HDAC9/microRNA-138/Sirtuin-1 Pathway Mediates Synaptic and Amyloid Precursor Protein Processing Deficits in Alzheimer's Disease. Neurosci Bull. 2019;35:87788.

22. Dube U, Del-Aguila JL, Li Z, Budde JP, Jiang S, Hsu S, Ibanez L, Fernandez MV, Farias F, Norton J, Gentsch J, Wang F, Salloway S, Masters CL, Lee JH, Graff-Radford NR, Chhatwal JP, Bateman RJ, Morris JC, Karch CM, Harari O, Cruchaga C. An atlas of cortical circular RNA expression in Alzheimer disease brains demonstrates clinical and pathological associations. Nat Neurosci. 2019;22:190312.

23. Peng P, Zhang B, Huang J, Xing C, Liu W, Sun C, Guo W, Yao S, Ruan W, Ning G, Kong X, Feng S. Identification of a circRNA-miRNA-mRNA network to explore the effects of circRNAs on pathogenesis and treatment of spinal cord injury. Life Sci. 2020;257:118039.

24. Lee EK, Jeong HO, Bang EJ, Kim CH, Mun JY, Noh S, Gim JA, Kim DH, Chung KW, Yu BP, Chung HY. The involvement of serum exosomal miR-500-3p and miR-770-3p in aging: modulation by calorie restriction. Oncotarget. 2018;9:5578-87.

25. Wang L, Li H. MiR-770-5p facilitates podocyte apoptosis and inflammation in diabetic nephropathy by targeting TIMP3, Biosci Rep, 40 (2020).

26. Qu W, Li L. Loss of TREM2 Confers Resilience to Synaptic and Cognitive Impairment in Aged Mice. J Neurosci. 2020;40:9552-63.

27. Wang $Y$, Lin $Y$, Wang $L$, Zhan $H$, Luo X, Zeng $Y$, Wu W, Zhang $X$, Wang F. TREM2 ameliorates neuroinflammatory response and cognitive impairment via PI3K/AKT/FoxO3a signaling pathway in Alzheimer's disease mice. Aging. 2020;12:20862-79.

28. Qin C, Fan WH, Liu Q, Shang K, Murugan M, Wu LJ, Wang W, Tian DS, Fingolimod Protects Against Ischemic White Matter Damage by Modulating Microglia Toward M2 Polarization via STAT3 Pathway, Stroke, 48 (2017) 3336-3346.

29. Tian M, Qingzhen L, Zhiyang Y, Chunlong C, Jiao D, Zhang L, Li W. Attractylone attenuates sepsisassociated encephalopathy and cognitive dysfunction by inhibiting microglial activation and neuroinflammation, J Cell Biochem, (2019).

30. Lu Y, Dong Y, Tucker D, Wang R, Ahmed ME, Brann D, Zhang Q. Treadmill Exercise Exerts Neuroprotection and Regulates Microglial Polarization and Oxidative Stress in a StreptozotocinInduced Rat Model of Sporadic Alzheimer's Disease. J Alzheimers Dis. 2017;56:1469-84.

\section{Figures}


A

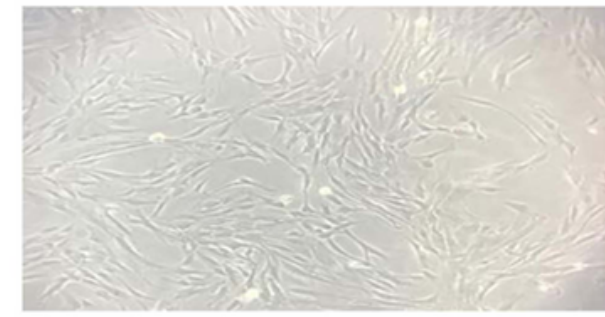

D

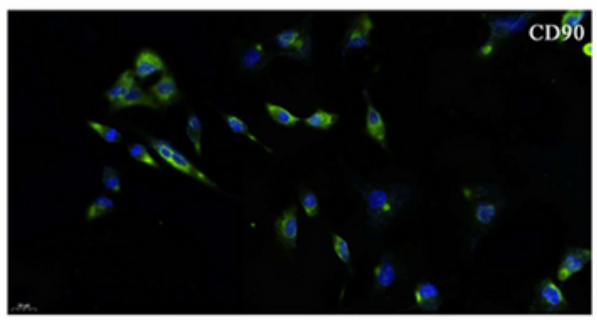

G

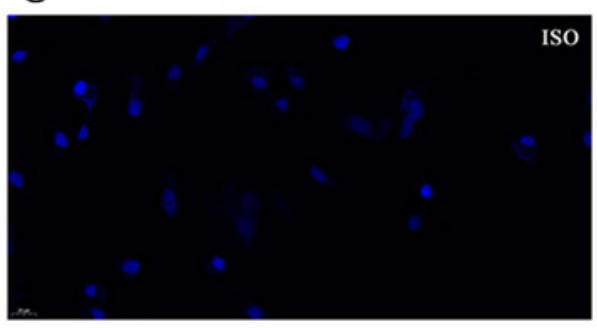

J
B

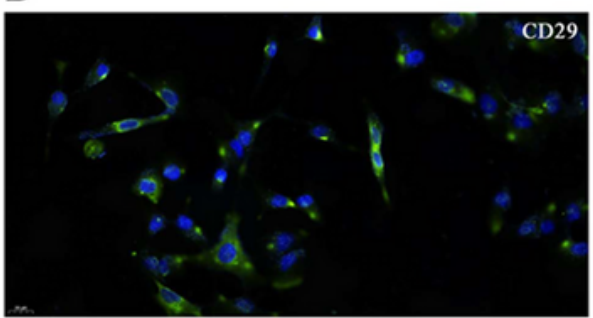

E

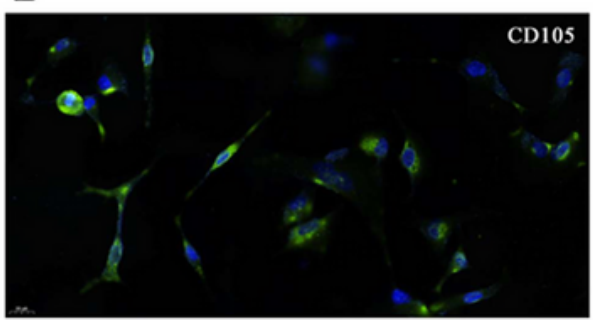

$\mathrm{H}$

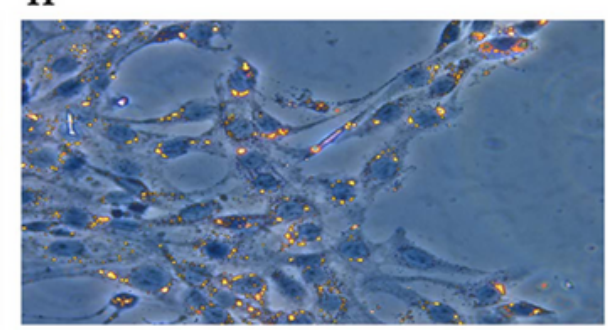

K

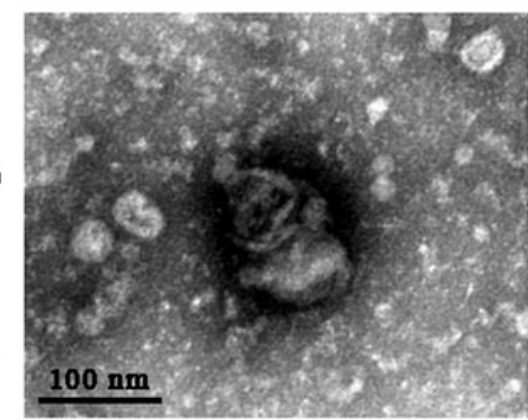

$43 \mathrm{kDa}$

CD63

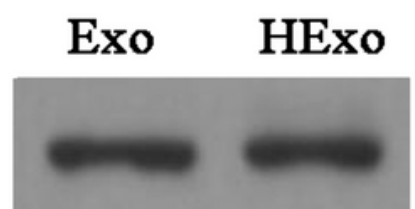

Exo

\section{CD9}

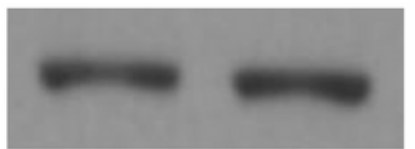

\section{$25 \mathrm{kDa}$}

\section{HExo}

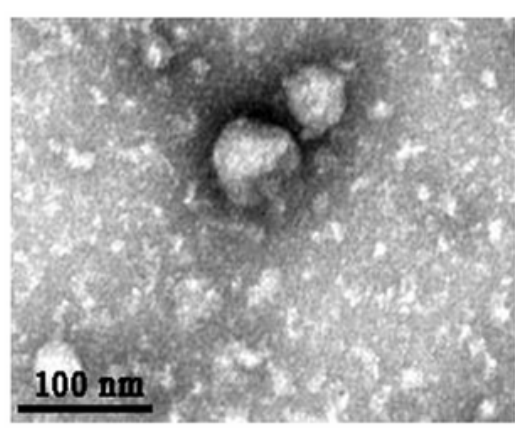

C

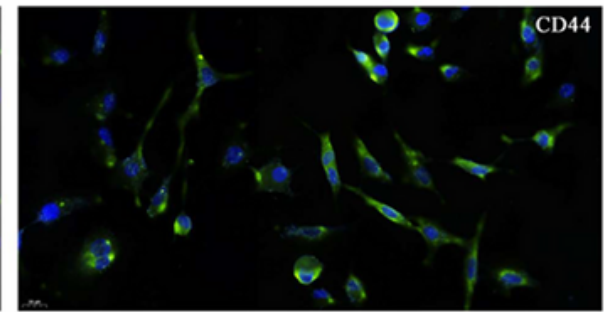

$\mathrm{F}$

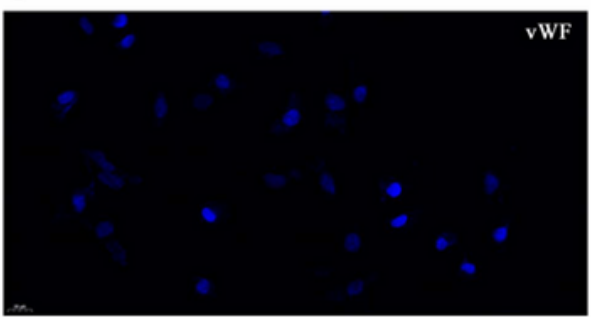

I

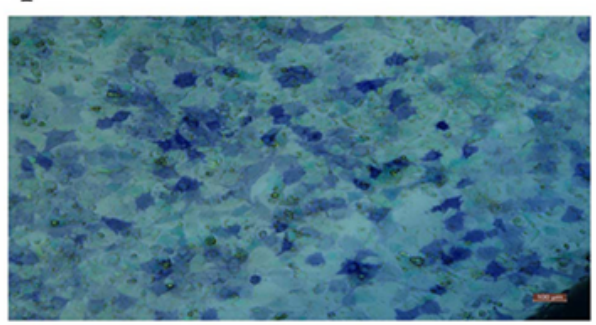

L $18.00 \mathrm{E}+10$

$16.00 \mathrm{~B}+10$

$14.00 \mathrm{E}+10$

$12.00 \mathrm{E}+10$

$10.00 \mathrm{E}+10$

$8.00 \mathrm{~B}+10$

$6.00 \mathrm{~B}+10$

$4.00 \mathrm{E}+10$

$2.00 \mathrm{E}+10$

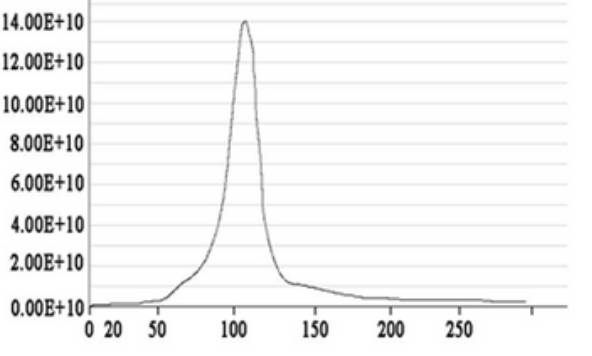

18. $00 \mathrm{E}+10$

$16.00 \mathrm{E}+10$

$14.00 \mathrm{E}+10$

$12.00 \mathrm{E}+10$

$10.00 \mathrm{E}+10$

$8.00 \mathrm{E}+10$

$6.00 \mathrm{~B}+10$

$4.00 \mathrm{~B}+10$

$2.00 \mathrm{E}+10$

$0.00 \mathrm{E}+10$

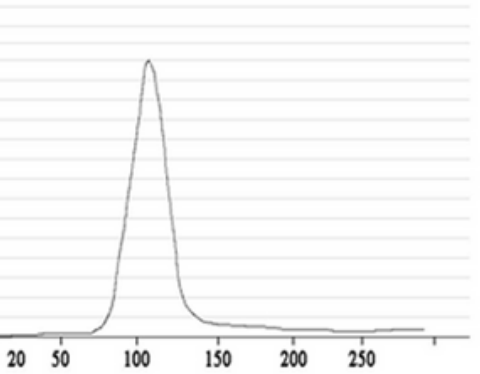

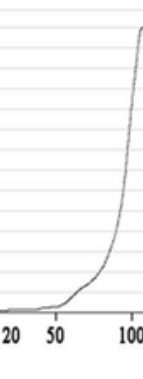

\section{Figure 1}

Characterization of exosomes released by adipose-derived mesenchymal stem cells (ADSCs). (A) ADSCs showed a typical cobblestone-like morphology. Scale bar: $30 \mu \mathrm{m}$. (B-G) Immunofluorescence staining of cell surface markers. The antibodies were labeled with fluorescein isothiocyanate (FITC, green). CD29, CD90, CD44, and CD105 were positive. von Willebrand Factor (vWF) was negative. FITC- and PE-labeled mouse IgG isotype controls are shown (magnification: 200x). Scale bar: $30 \mu \mathrm{m}$. (H and I) Differentiation 
potential of ADSCs assessed by oil red $\mathrm{O}(\mathrm{H})$ and alkaline phosphatase staining (I). Scale bar: $50 \mu \mathrm{m} .(\mathrm{J})$ Western blots of CD63 and CD81 expression in exosomes from hypoxia-pretreated or WT ADSCs. (K) Transmission electron micrographs showing ADSC-exosome morphology. Scale bar: $100 \mathrm{~nm}$. (L) Particle size distribution and concentration of ADSC-exosomes measured by nanoparticle tracking analysis.

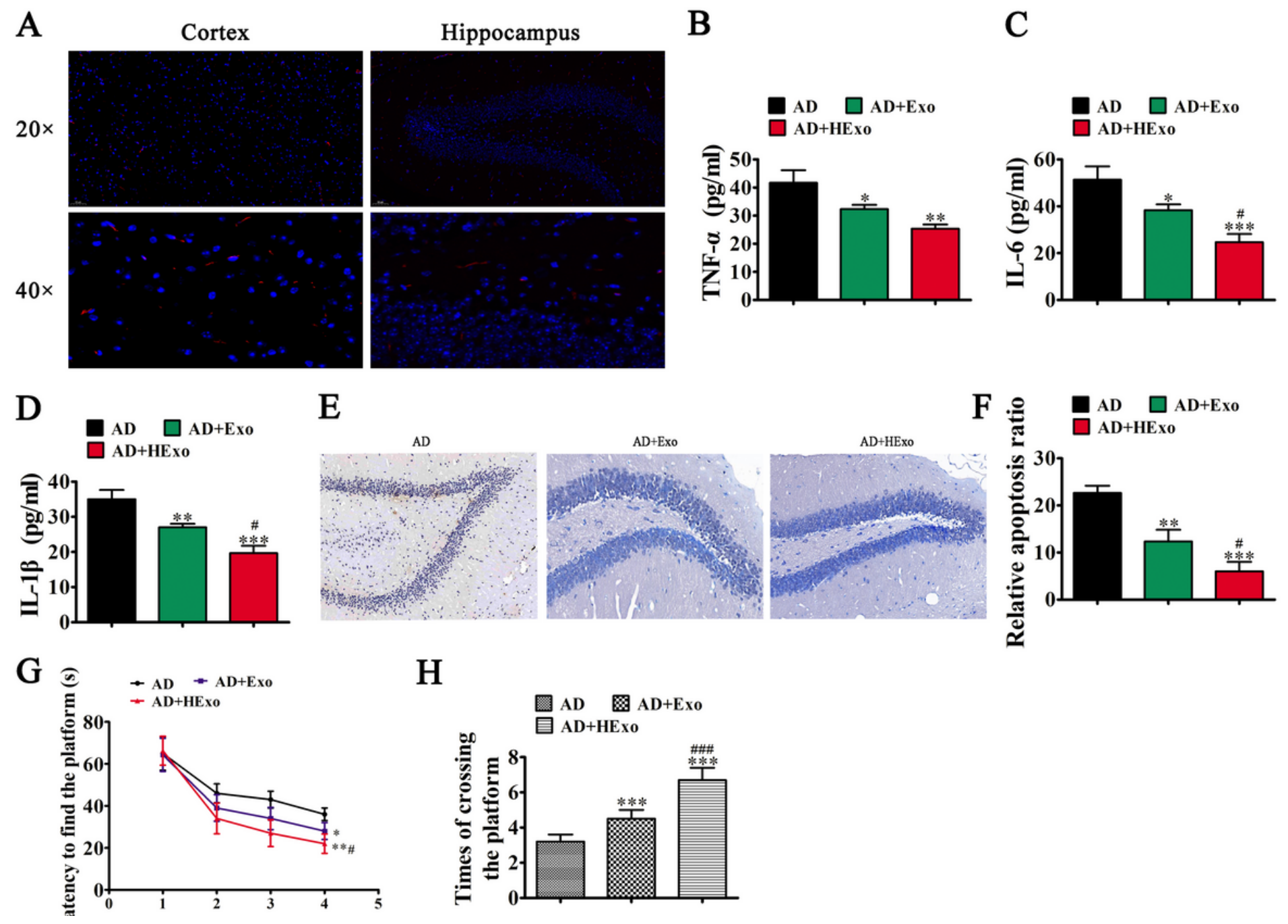

Figure 2

Exosomes from hypoxia-pretreated ADSCs have more therapeutic effect at improving cognitive function by decreasing neuronal damage in the hippocampus. (A) Dil-labeled exosomes are red and nuclei are counterstained with DAPI (blue). The injected exosomes were detected in the cortex and hippocampus. (B-D) ELISA detection showing expression of the inflammatory factors TNF- $\alpha$, IL-6, and IL-1 $\beta$. Data represent mean $\pm S D(n=10) .{ }^{*} P<0.05,{ }^{\star} * P<0.01$, ${ }^{\star \star \star} P<0.001$ vs. control; $\# P<0.05$ vs. Exo. (E and $\left.F\right)$ Hippocampal neuron apoptosis was detected via the TUNEL assay. Data represent mean $\pm S D(n=6)$. **P $<0.01,{ }^{*} * \mathrm{P}<0.001$ vs. control; $\# \mathrm{P}<0.05$ vs. Exo. (G) $A D$ mice exhibited a longer escape latency than exosome-treated animals. Data represent mean $\pm S D(n=10)$. ${ }^{*} P<0.05,{ }^{*} P<<0.01$ vs. control; $\# P<0.05$ vs. Exo. $(H)$ The number of platform crossings was increased in the exosome-treated group. Data represent mean $\pm S D(n=10) .{ }^{\star} P<0.05,{ }^{\star} * \star P<0.001$ vs. control; \#\#\#P<0.001 vs. Exo. 
A

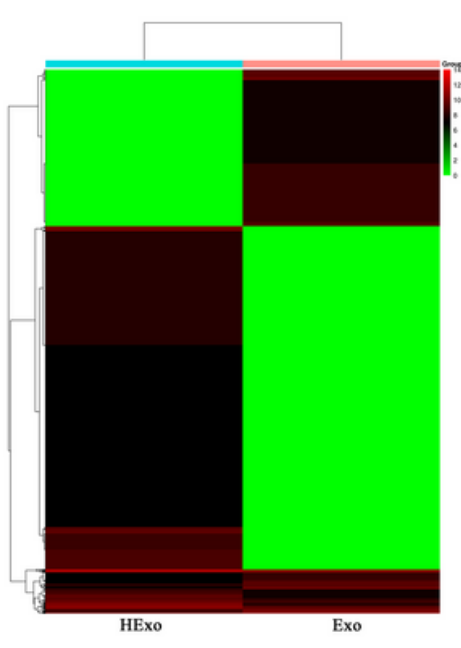

B

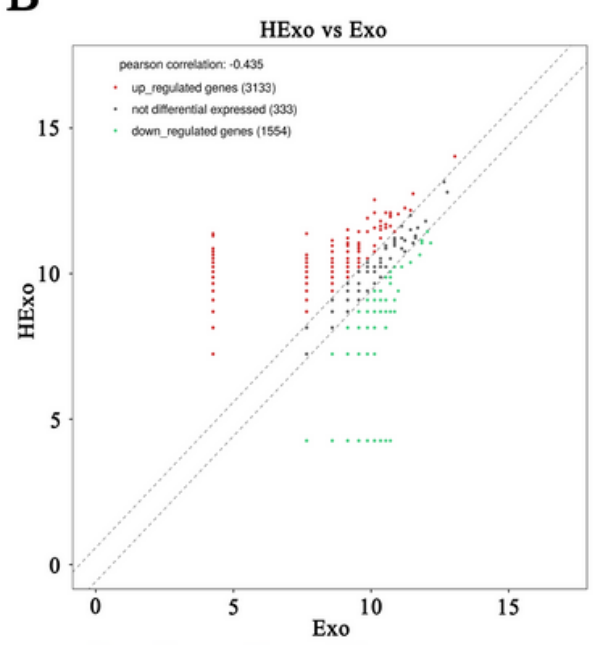

C

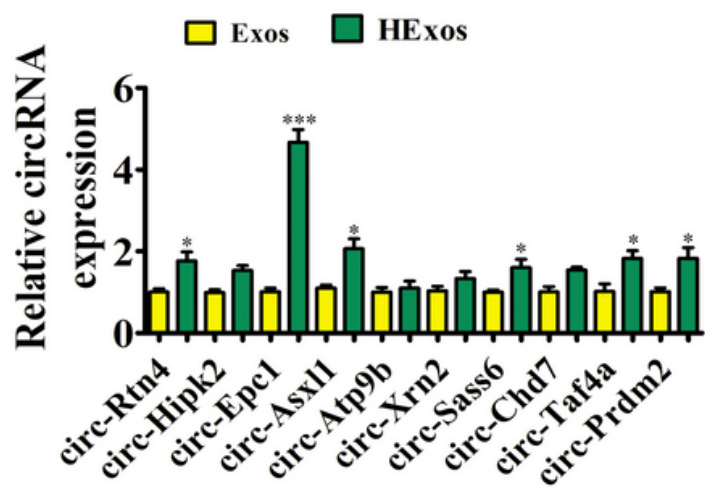

D

\section{Color key for alignment scores}

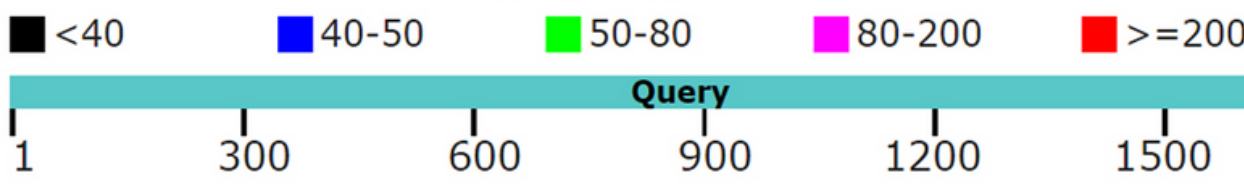

\section{1}

chr18:6448902-6450637

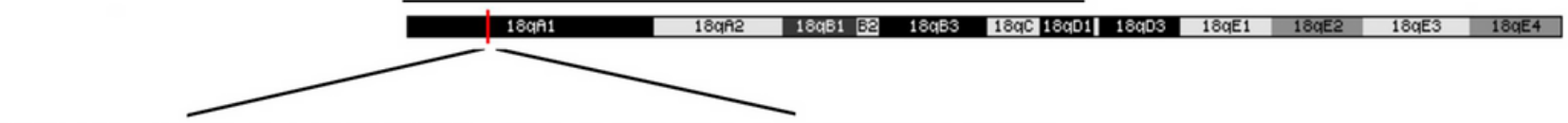

CTCAGCACACTGTG CAAGATAAGGCTGATC

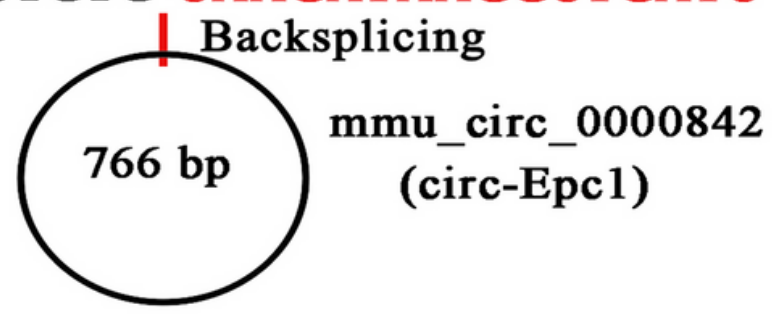

Figure 3

Exosomes from hypoxia-pretreated ADSCs have a greater therapeutic effect at improving cognitive function when delivering circ-Epc1. (A) Heatmap showing the differentially expressed circRNAs. (B) Volcano Plot showing the upregulated and downregulated circRNAs from Exo and HExo. (C) RT-qPCR detection showing the expression of 10 upregulated circRNAs between Exo and HExo. Data are means \pm SD. ${ }^{*} P<0.05,{ }^{\star} * \star \mathrm{P}<0.001$ vs. Exo. (D) The chromosomal location of circ-Epc1. 
A

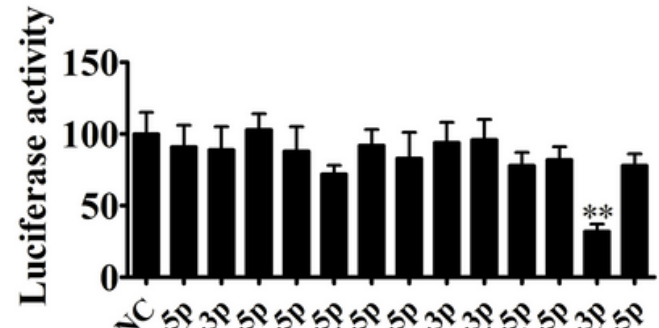

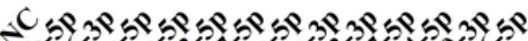

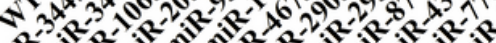

D

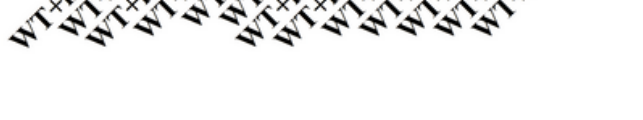

TREM2 3' UTR-WT $\quad 5^{\prime} \quad$ cegeCUCUGCCUCAAGCCCACc $3^{\prime}$ | ||:: | | || ||||||

TREM2 3' UTR-Mut $\quad 5^{\prime} \quad$ ecgcCUCUGCCUCAAAAGGACc $3^{\prime}$

\section{G}

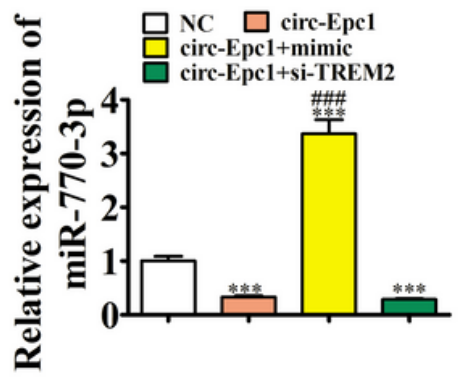

B

circ-Epc1-WT $\quad 5^{\prime} \quad$ geAGCACAGCCUGGGGCCCACa $3^{\prime}$ || | : | | : || || || | miR-770-3p $\quad 3^{\prime}$ ggUCGAGGUGCAGUCCGGGUGc 5' circ-Epc1-Mut $\quad 5^{\prime}$ gcAGCACAGCCUGGAAGGCACa 3'

$\mathrm{E}$

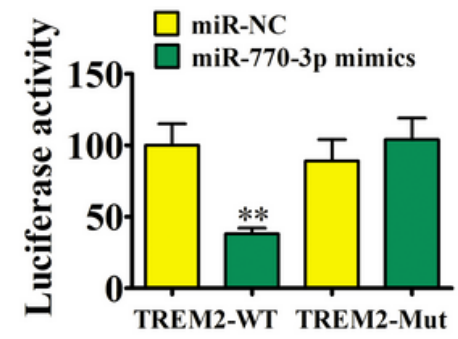

$\mathrm{H}$

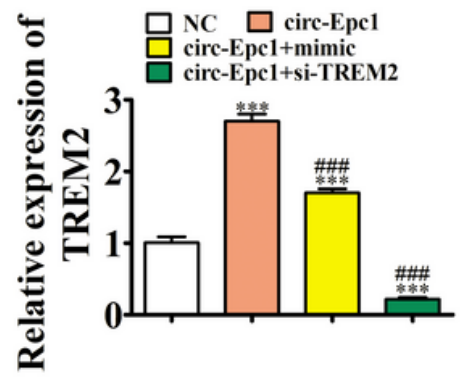

C
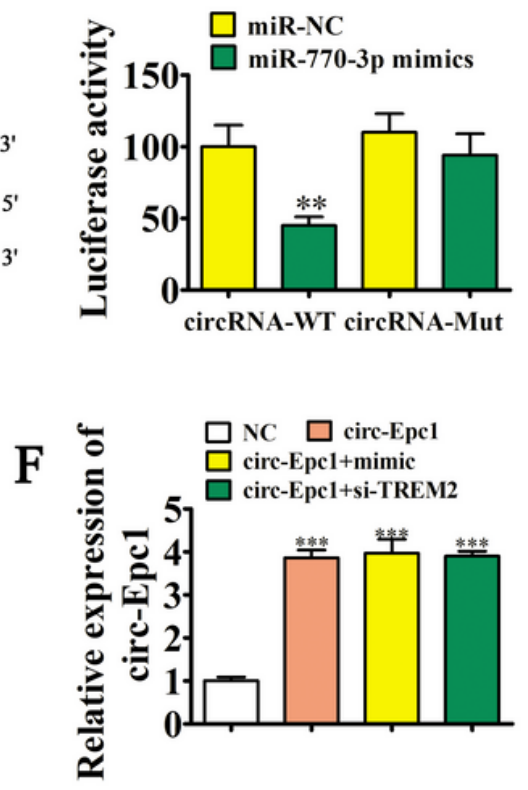

Figure 4

TREM2 and miR-770-3p are downstream targets of circ-Epc1. (A) Dual-luciferase reporter assay showed that co-transfecting WT and mimic miR-770-3p markedly decreased luciferase activity in HEK293T cells. Data are means \pm SD. ${ }^{*} \mathrm{P}<0.01$ vs. WT+NC. (B) Predicted binding sites of miR-770-3p in circ-Epc1. The mutant version of circ-Epc1 is presented. (C) Relative luciferase activity determined $48 \mathrm{~h}$ after transfecting HEK293T cells with miR-770-3p mimic/NC or circ-Epc1 WT/Mut. Data are presented as means $\pm S D$. ${ }^{*} P<0.01$. (D) Predicted binding sites of miR-770-3p within the 3 '-UTR of TREM2. The mutant version of the TREM2 3 '-UTR is shown. (E) Relative luciferase activity determined $48 \mathrm{~h}$ after transfecting HEK293T cells with miR-770-3p mimic/NC or 3'-UTR-TREM2 WT/Mut. Data are presented as means \pm SD. ${ }^{* *} \mathrm{P}<0.01$. (F-H) RT-qPCR detection showing the expression of circ-Epc1, miR-770-3p, and TREM2 in BV2 cells after transfection with circ-Epc1 overexpression (circ-Epc1), miR-770-3p mimic (mimic), TREM2 silencing vector (si-TREM2) individually or in combination. Data are presented as means \pm SD. $* \star * P<0.001$ vs. NC; \#\#\#P $<0.001$ vs. circ-Epc1. 
$\mathbf{A}$

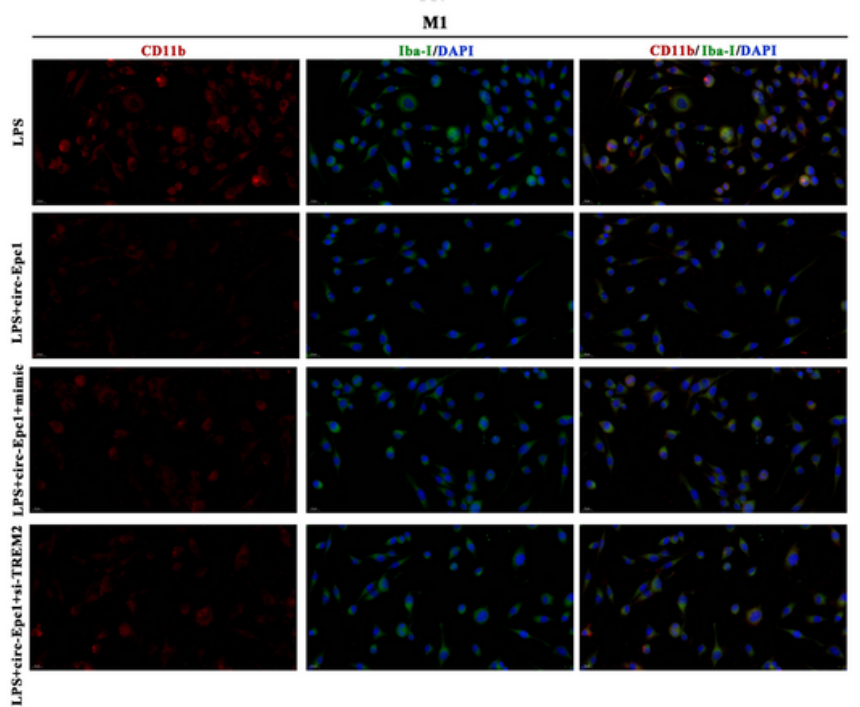

$\mathbf{C}$

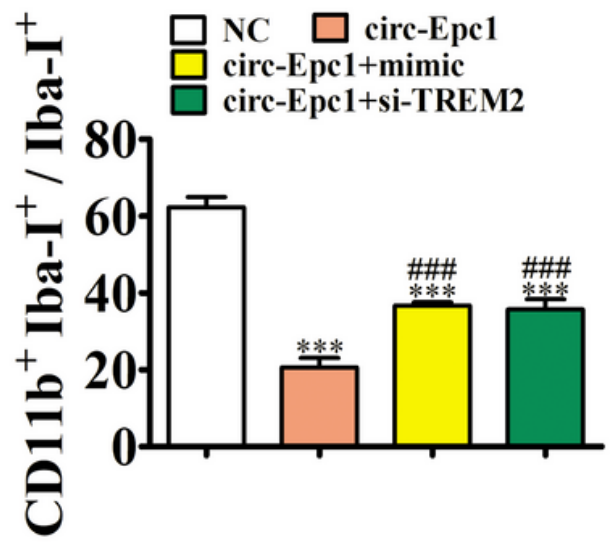

B

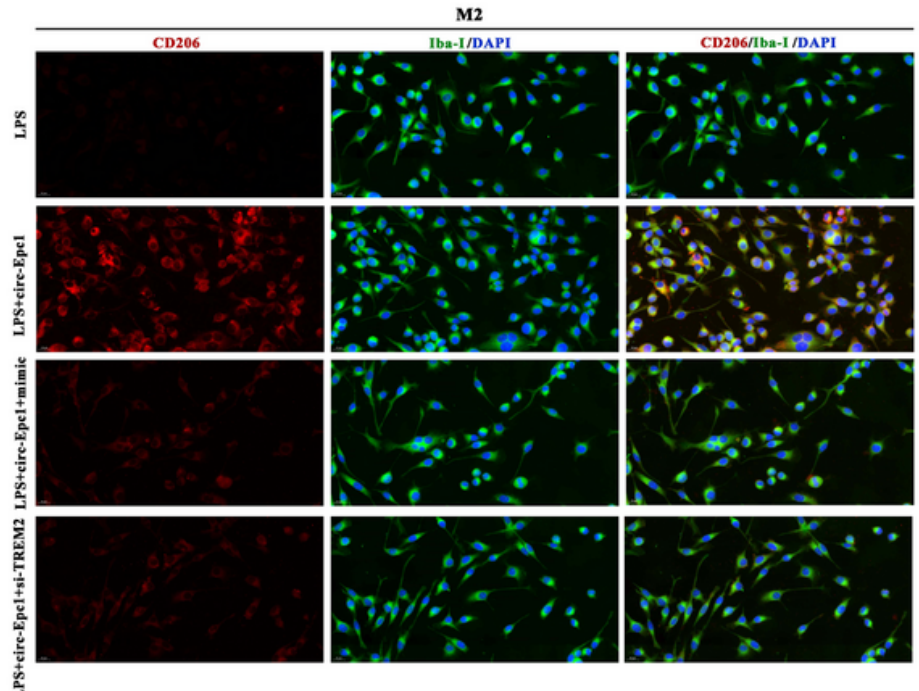

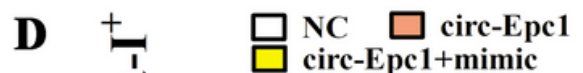

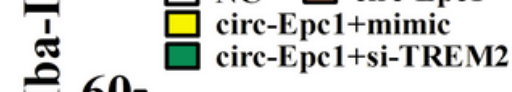

ปั

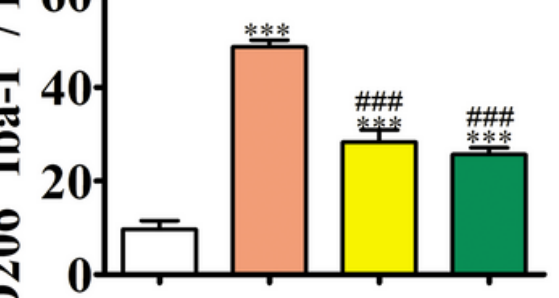

$\mathbf{E}$

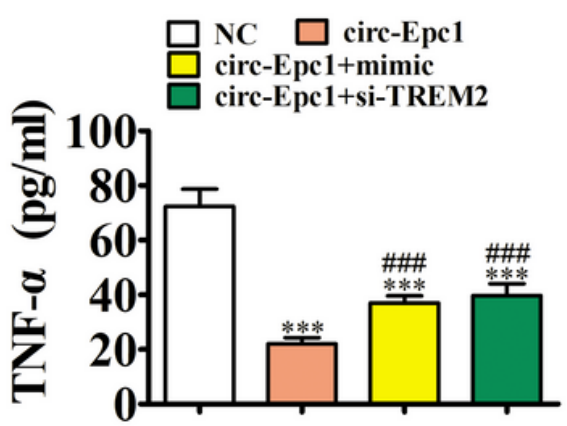

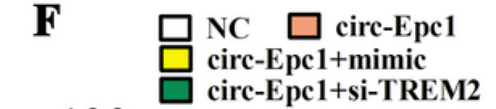

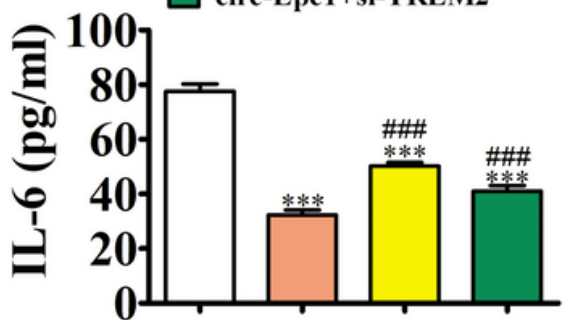

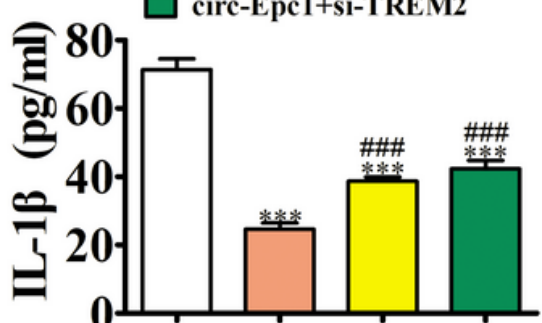

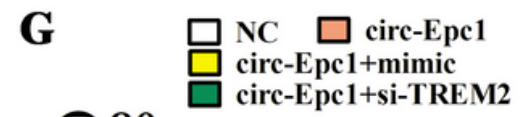

Figure 5

Overexpressing miR-770-3p or downregulating TREM2 reversed the effect of circ-Epc1 on M2 microglial shifting under LPS $(1 \mu \mathrm{g} / \mathrm{mL})$. (A-D) Immunofluorescence detection of macrophage polarization using

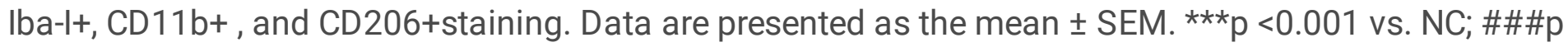
$<0.001$ vs. circ-Epc1. (E-G) ELISA detection showing expression of the inflammatory factors TNF-a, IL-6, and IL-1 $\beta$. Data are presented mean \pm SD $(n=10)$. ${ }^{* \star P} P<0.001$ vs. NC; \#P $<0.05$ vs. circ-Epc1. 
A

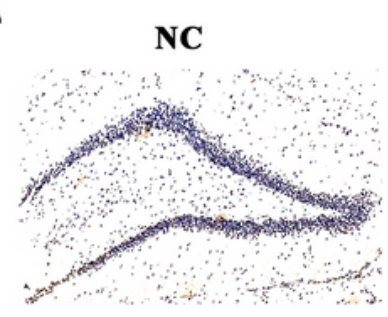

C

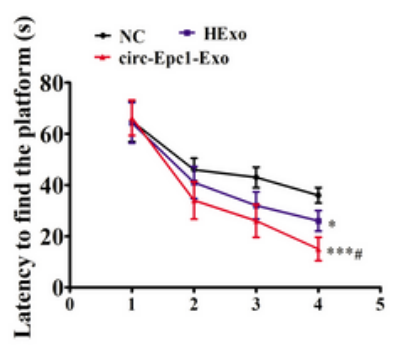

F

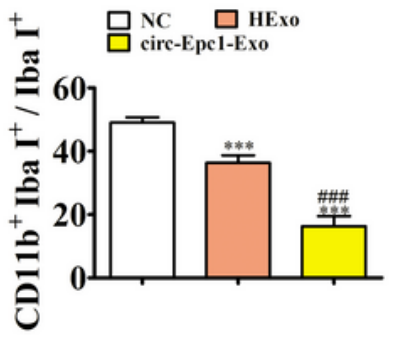

HExo

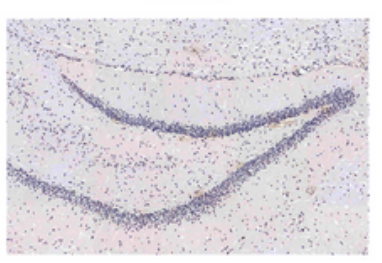

D

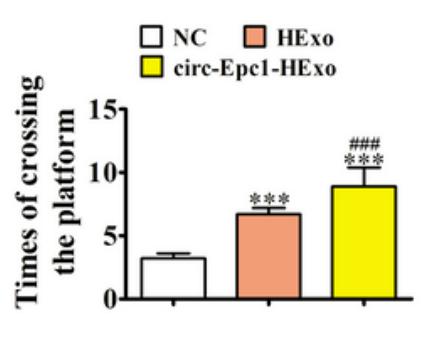

G

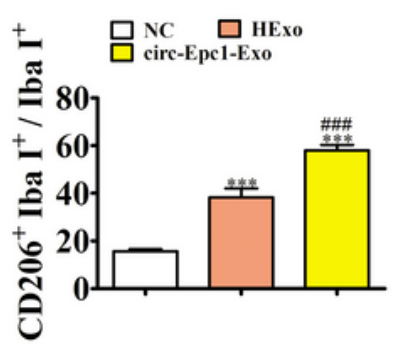

H
circ-Epc1-Exo

\section{B}

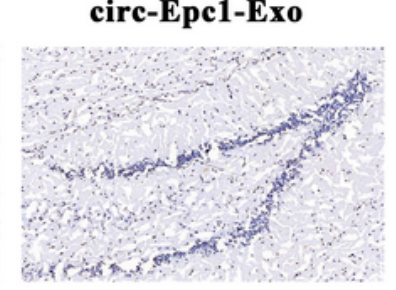

윯 $\square$ circ-Epe1-Exo

$\mathbf{E}$
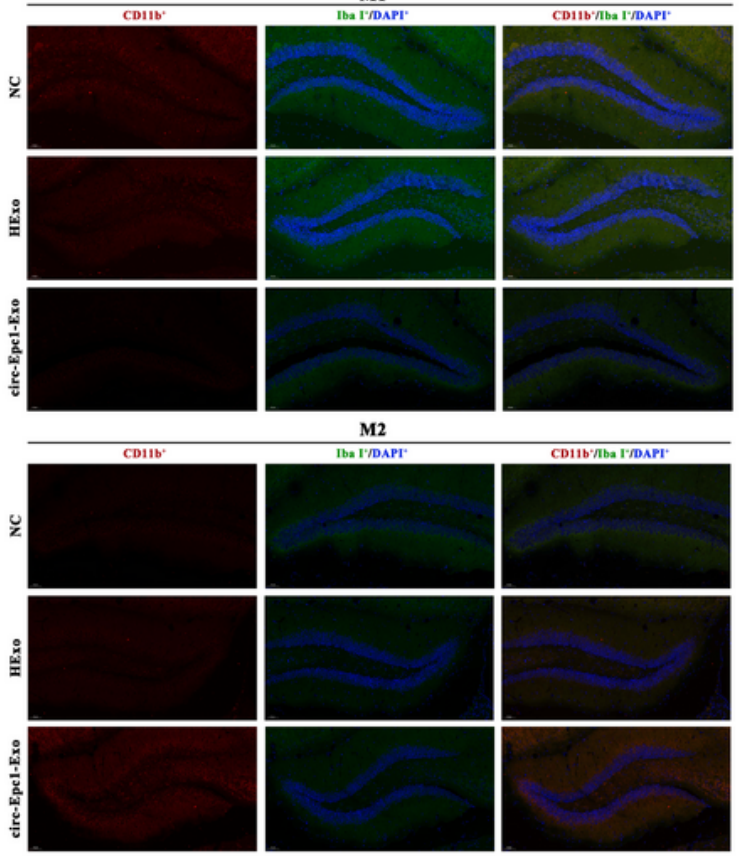

M2
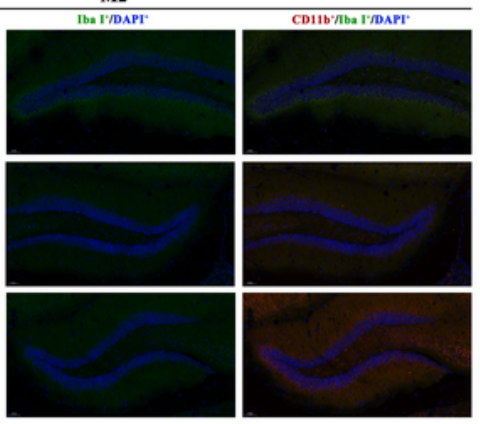

I

J
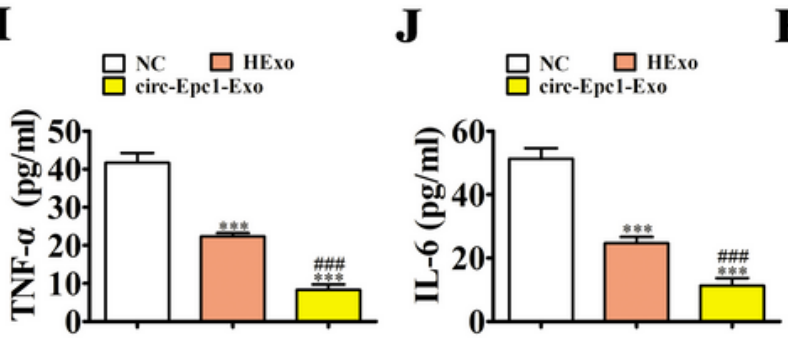

K

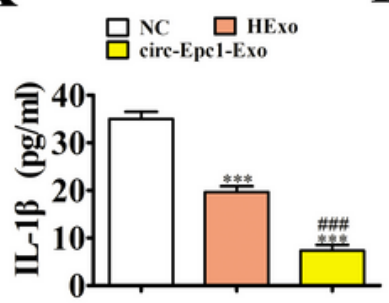

L

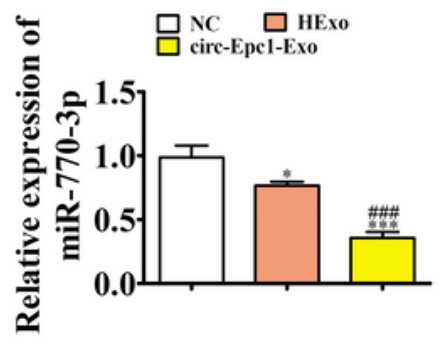

M

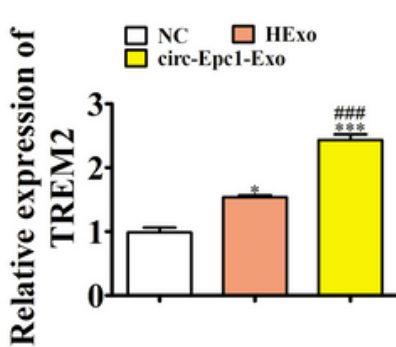

\section{Figure 6}

Circ-Epc1-modified ADSC exosomes (circ-Epc1-Exo) showed increased therapeutic effects at improving cognitive function by decreasing neuronal damage and shifting hippocampal microglia from M1 to M2. (A and B) Hippocampal neuron apoptosis was detected with the TUNEL assay. Data represent mean \pm SD $(n=6) .{ }^{\star} \mathrm{P}<0.01,{ }^{\star} * \star \mathrm{P}<0.001$ vs. control; $\# P<0.05$ vs. Exo. (C) AD mice exhibited a longer escape latency than exosome-treated animals. Data represent mean $\pm S D(n=10)$. ${ }^{*}<<0.05$, ${ }^{*} P<<0.01$ vs. control; \#P 
$<0.05$ vs. HExo. (D) The number of platform crossings was increased in the exosome-treated group. Data represent mean $\pm S D(n=10) .{ }^{*}<0.05, * \star * P<0.001$ vs. control; \#\#\#P<0.001 vs. HExo. $(E-H)$ Immunofluorescence detection of macrophage polarization using $\mathrm{F} 4 / 80+, \mathrm{CD} 11 \mathrm{~b}+$, and CD206+staining. Data are presented as the mean \pm SEM. ${ }^{\star * \star} p<0.001$ vs. control; \#\#\#p $<0.001$ vs. HExo. (I-K) ELISA detection showing expression of the inflammatory factors TNF-a, IL-6, and IL-1 $\beta$. Data are presented as the mean \pm SEM. ${ }^{* \star *} p<0.001$ vs. control; \#\#\#p <0.001 vs. HExo. ( $L$ and $\left.M\right) R T-q P C R$ detection showing the expression of miR-770-3p and TREM2 in hippocampal tissues. Data are presented as the mean \pm SEM. ***p $<0.001$ vs. control; \#\#\#p <0.001 vs. HExo. 